\title{
Traduire
}

Une eutre perspective sur $r$ tatadciction

Revue française de la traduction

$240 \mid 2019$

Quand la politique s'en mêle

\section{Discours politique et récit national}

Sabrina Ariès Lardeux

\section{OpenEdition}

Journals

Édition électronique

URL : http://journals.openedition.org/traduire/1640

DOI : 10.4000/traduire.1640

ISSN : 2272-9992

Éditeur

Société française des traducteurs

Édition imprimée

Date de publication : 20 juin 2019

Pagination : 15-23

ISSN : 0395-773X

\section{Référence électronique}

Sabrina Ariès Lardeux, « Discours politique et récit national », Traduire [En ligne], 240 | 2019, mis en ligne le 20 juin 2019, consulté le 06 février 2020. URL : http://journals.openedition.org/traduire/1640 ; DOI : 10.4000/traduire.1640 


\section{Discours politique et récit national}

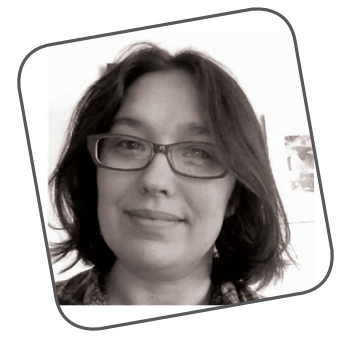

Sabrina Ariès Lardeux

En ces temps de crise des vieux États-nations, le récit national s'invite bien souvent dans le discours politique, soit pour préciser le contour de frontières perçues comme floves, soit pour exalter et défendre une identité nationale prétendument menacée. Loin du traducteur l'idée de juger ce recours aux mythes fondateurs convoqués pour rassembler un peuple autour d'un même récit. La question est plus ardue lorsqu'il s'agit de le retranscrire dans le cadre de relations diplomatiques ou pour la presse. Outre les références culturelles, sociales et historiques, les connotations et les euphémismes présents dans le discours politique témoignent d'une élasticité des concepts qu'il n'est pas toujours aisé de traduire - et cela davantage encore dans le discours nationalnationaliste conçu pour exalter le sentiment d'appartenance à une communauté d'esprit et de destin. C'est précisément le caractère mouvant - et parfois glissant, voire ambigu - des termes utilisés dans ce type de discours qui le rend si délicat à traduire. Qu'il s'adresse à une frange restreinte de la population ou qu'il vise à toucher l'audience la plus large possible, le discours national-nationaliste use toujours de l'art d'écrire les fluctuations de son propre récit historique. Cette ambiguïté obligera bien souvent le traducteur à nager dans les eaux troubles de la nuance et de l'interprétation, tout en s'efforçant d'analyser, avec la distance adéquate et sans s'y noyer, les actes et les contextes 
de communication, ainsi que les rôles et les comportements des locuteurs et des destinataires. À propos de la notion d'ambiguïté du discours diplomatique, Olivier Arifon indique:

Dans la langue française, un grand nombre d'expressions montrent la richesse de cette notion: ambages, allusions, demi-mots, contenus latents, sens cachés, sous-entendus, arrière-pensées, etc. Les procédures de langage, leurs compréhensions, en un mot l'analyse des situations du discours, des silences et des affirmations, sont bien une aptitude indispensable au métier de diplomate.

Remplaçons diplomate par traducteur et le tour est joué: voilà le portrait-robot du parfait traducteur de discours politique et/ ou diplomatique! Même si cela semble une évidence, celui-ci doit, pour mener sa tâche à bien, se défaire de ses oripeaux culturels, historiques et politiques pour parvenir à se mettre à égale distance du locuteur et du destinataire. Une lapalissade qui se transforme parfois en gageure.

Pour illustrer mon propos, je m'appuierai en particulier sur la "crise catalane» et les stratégies de communication politique mobilisées par le gouvernement catalan pour promouvoir la cause indépendantiste par-delà ses frontières, notamment en France et en Belgique, depuis 2010 et plus intensément depuis le 1er octobre 2017. De prime abord, la tradition jacobine française et la définition de la nation qui en découle rendent l'existence d'une nation catalane au sein de l'Espagne difficilement audible. Nous verrons que le récit national, par sa spécificité, complique la transposition des éléments de langage du discours nationaliste d'une langue à l'autre. Le choix des termes revêt un caractère essentiel lorsqu'il s'agit de sensibiliser un auditoire à une cause, quelle qu'elle soit. Le procés indépendantiste catalan ne déroge pas à la règle, émaillant ses discours de références à la lutte pour les droits civiques dans les États-Unis des années 1950 1960, à la répression de la dictature franquiste, à la Déclaration universelle des droits de l'homme ou encore à la révolution ukrainienne de 2014, pour tenter d'entrer en résonance avec une mémoire universelle plutôt que catalano-centrée. Lorsque les mots "démocratie», "peuple», "nation», "prisonniers politiques», "répression» sont agités comme des chiffons rouges dans les discours, quel espace existe-t-il pour le traducteur, devenu jongleur (ou funambule, c'est selon) sur la piste des 
éléments de langage, de la langue de bois et autres novlangues du cirque politico-médiatique?

\section{Le récit national, entre histoire intime et identité collective}

Avant d'entrer dans le vif du sujet, je me dois de répondre à deux questions: qu'est-ce qui distingue ce que l'on nomme le récit national de tout autre type de discours politique? En quoi cela affecte-t-il particulièrement l'acte de traduire?

Dans la tradition historiographique française héritée du $X I X^{e}$ siècle, dont les figures tutélaires sont Ernest Lavisse et Jules Michelet, le récit national (voire le «roman national») se conçoit comme un récit patriotique et centralisateur, tout à la louange de la nation, avec ses héros et ses hauts faits d'armes, tout autant science que liturgie. II suffit de relire la couverture du manuel d'histoire de France à l'attention du cours moyen rédigé par Lavisse (édition de 1913) pour saisir l'ampleur de cette mission politique:

Enfant, tu vois sur la couverture de ce livre les fleurs et les fruits de la France. Dans ce livre, tu apprendras l'histoire de la France. Tu dois aimer la France, parce que la nature l'a faite belle et parce que son histoire l'a faite grande.

Pour Patrick Boucheron et Nicolas Delalande, auteurs avec un collectif de 122 historiennes et historiens d'une Histoire mondiale de la France (Éditions du Sevil, 2017) cherchant à offrir une vision alternative de l'histoire qui soit dégagée du parti pris du roman national trop souvent exploité par le discours politique,

[I]'histoire des appartenances montre en effet que l'identification nationale ne s'impose qu'au XIXe siècle, à travers des dispositifs, des discours ou des politiques publiques dont les historiennes et les historiens se font les analystes minutieux.

Ainsi, le récit national s'élabore à partir de vérités énoncées par certains historiens soucieux de surligner les évènements fondateurs, unificateurs - et si possible glorificateurs - de leur pays. Il est repris à l'envi par des hommes et des femmes politiques de tout bord utilisant l'histoire pour justifier une posture morale ou politique. Preuve de l'importance de l'enjeu politique du récit historique et national, et à la suite du succès remporté 
par l'entreprise de Patrick Boucheron, on verra d'ailleurs apparaître en Espagne une Historia mundial de España, sous la direction de Xosé M. Nuñez Seixas (Ediciones Destino, 2018), et, en Catalogne, une História mundial de Catalunya, sous la direction de Borja de Riquer (Ediciones 62, 2018).

Dans le contexte catalan, le discours national-nationaliste recourt abondamment au récit historique pour fédérer le peuple autour du projet indépendantiste, à l'instar des propos tenus par Artur Mas, alors président de la Généralité de Catalogne, lors de son allocution du 27 septembre 2014 - après la signature du décret convoquant la consultation du 9 novembre 2014 sur l'avenir politique de la Catalogne:

Tengo el honor de ser el129 presidente de la Generalitat de Cataluña. institución creada en 1359 y que desde la primera presidencia de Berenguer de Cruilles ha reflejado durante casi siete siglos la voluntad de autogo bierno de los catalanes. A lo largo de estos siete siglos solamente imposiciones externas han provocado la suspensión del autogobierno. Un autogobierno que la voluntad expresa del pueblo catalán siempre ha deseado recuperar. Nuestras raices son profundas. como lo es la solidez de nuestro sentimiento. Pero más sólidas resultan todavia la voluntad y la determinación de construir un pais mejor de cara al futuro. Un buen pais.
J'ai l'honneur d'être le $129^{\circ}$ président de la Généralité de Catalogne, institution créée en 1359 et qui, depuis la première présidence de Berenguer de Cruïlles, a affirmé pendant près de sept siècles la volonté des Catalans de se gouverner eux-mêmes. Au cours de ces sept siècles, le gouvernement autonome n'a dû sa suspension qu'à des contraintes extérieures. Un gouvernement autonome que la volonté expresse du peuple catalan a toujours désiré retrouver. Nos racines sont aussi profondes que notre sentiment est solide. Mais plus solides encore sont notre volonté et notre détermination à construire un meilleur pays pour l'avenir. Un bon pays.

De son côté, l'historien Jaume Sobrequés i Callicó explique dans l'ouvrage História de Catalunya que son travail répond tout autant à «une intention scrupuleusement rigoureuse» qu' à "une motivation sociale et politique», ainsi qu'à "une volonté inébranlable de servir le pays».

On l'aura compris, cette invitation incessante de l'histoire dans le discours politique et cette irruption du récit national dans le champ de la parole publique représentent un enjeu de taille pour le traducteur: comment se défaire de son propre bagage historico-politico-culturel (conscient ou inconscient) pour ne pas tomber dans les écueils de la surinterprétation? Et comment 
transcrire la part d'inconscient collectif culturel et émotionnel du discours national?

\section{Les métamorphoses du discours}

Après «l'intensive opération nationalisatrice, voire renationalisatrice, de la société catalane» du pujolisme (Jordi Pujol a été président de la Généralité de Catalogne de 1980 à 2003), bien détaillée par Jordi Canal dans un ouvrage publié peu de temps après le référendum d'autodétermination du $1^{\text {er }}$ octobre 2017, le discours nationaliste a entamé il y a plusieurs années une nouvelle étape d'extériorisation de la cause qu'il défend sur la scène internationale, à savoir le droit à l'autodétermination de la nation catalane. Cette divulgation, portée par une campagne de lobbying auprès de différents organismes et institutions européens, conduit à l'adoption des codes du discours diplomatique, plus neutre et formel.

Il ne s'agit plus d'exalter le sentiment d'appartenance à la nation catalane (avec succès depuis plus de trente ans, d'après Jordi Canal), mais bien plutôt de justifier - vis-à-vis de l'extérieur - et de légitimer une communauté de destin dans l'exercice de son droit fondamental d'exister et de choisir. Si je donne cette précision, ce n'est pas tant pour le plaisir de décortiquer et de gloser sur les stratégies de communication politique mises en œuvre dans le discours indépendantiste catalan, que pour montrer que le destinataire ayant changé, son objectif n'est plus le même et partant, la charge du propos en est transformée.

Pour montrer que le "problème catalan» ne peut se réduire à une simple (et ô combien complexe) question de politique intérieure de l'État espagnol, mais qu'il relève d'une problématique éminemment européenne, les dirigeants indépendantistes doivent adopter un discours qui ne les situe plus seulement dans le champ intérieur et intime de l'identité, mais dans celui plus standardisé et formel des relations entre pays. Faire appel, en somme, à un langage commun, à une terminologie dont la puissance évocatrice transcende les frontières culturelles. Si leurs réalités et leurs manifestations peuvent différer, les termes "répression», "injustice», "démocratie» renvoient bon an mal an à une mémoire et à une compréhension communes. Pour être efficace, le discours doit ainsi adopter une approche stratégique dont l'objectif est de démultiplier son audience en procédant 
à un double mouvement de dilution et de radicalisation de la langue pour être compris de tous. Ainsi, dans son allocution de déclaration/suspension d'indépendance de la Catalogne sous la forme d'une République, Carles Puigdemont proclamait le 10 octobre 2017:

Vivim un moment excepcional. de dimensió històrica. Les seves conseqüències i efectes van molt més enllà del nostre pais i sha fet evident que. lluny de ser un afer domèstic i intern. com sovint hem hagut descoltar de part dels qui han negligit la seva responsabilitat per no voler conèixer el que estava passant. Catalunya és un afer europeu
Nous vivons un moment exceptionnel, historique. Ses conséquences et ses effets dépassent les frontières de notre pays, et il est aujourd'hui évident que, contrairement à ce que nous avons coutume d'entendre de la part de ceux qui négligent leur responsabilité en refusant de voir ce qui est en train de se produire, la Catalogne ne se limite pas à une question de politique interne, mais concerne bel et bien toute l'Europe.

Or, si l'on sait que l'une des prémisses à l'acte de traduire est de bien définir le locuteur et le destinataire, force est de constater que le destinataire n'est plus un, mais multiple. Nous vivons dans une époque où les actes de paroles - institutionnels ou non - sont amplifiés. Qu'un responsable politique publie une saillie sur Twitter, s'exprime au micro d'un journaliste au détour d'un couloir ou prononce un discours formel, ses mots se retrouveront propulsés sur des centaines de supports (réseaux sociaux, dépêches de presse), tronçonnés, décortiqués et commentés jusqu'à la lie. Cette situation modifie profondément la charge du discours et a entraîné l'émergence d'une culture du «clash»ou du «buzz» qui ne conçoit la prise de parole que comme une course au mieux-disant ou comme une parole plus vraie que la vérité elle-même. Pour Joanna Nowicki et Michaël Oustinoff,

[I] "langue de bois» est percue comme la langue d'hier, obsolète et ringarde. Elle est souvent remplacée aujourd'hui par le "parler vrai», une langue sans détour qui - contrairement à la langue de bois qui communique sans informer - informe en communiquant, directement dans la langue de l'autre, une langue simple, comprise par tous et non réservée à une élite. 
Dès lors, comment traduire la charge du discours tout en adoptant les codes du marketing politique, alliant tout à la fois paroles creuses et paroles crues?

Cette charge du discours repose sur ce que John R. Searle nomme les faits institutionnels:

Je propose d'appeler ces faits auxquels se rapporte le groupe d'affirmation mentionné plus haut, des faits institutionnels. II s'agit bien de faits, mais leur existence, à la différence des faits bruts, suppose l'existence de certaines institutions humaines. [...] C'est seulement parce qu'existe l'institution de la monnaie, que j'ai en ce moment dans la main un billet de cina dollars. Retirez l'institution, et je n'aurai plus qu'un morceau de papier sur lequel sont inscrites des choses en gris et vert.

Cependant, cette institution ne peut exister que si le contexte culturel, historique et social est défini. Dès lors que le destinataire est multiple et que les actes de langage sont polymorphes, que reste-t-il de la charge du discours, du signifiant et du signifié? Doit-on pour autant adopter les sacro-saints éléments de langage standardisé au bénéfice d'une lingua franca et au détriment de la part intraduisible de la charge du discours?

Cette question de la langue franque se pose dès lors qu'une déclaration est extraite de son contexte (culturel, historique, politique) et largement diffusée. Dans le discours national-nationaliste catalan, on a déjà eu l'occasion de relever de tels marqueurs d'ajustement. Si l'intervention de Carles Puigdemont se situait dans le registre des valeurs universelles (démocratie, droit à l'autodétermination des peuples, droits fondamentaux) adoptant le langage formel des relations internationales, d'autres éléments de langage introduits dans les prises de parole publique visent quant à eux à mobiliser une charge émotionnelle, un sens universellement reconnu et identifiable par tous. Prenons par exemple la référence aux drets civils ou aux drets civics. À de nombreuses reprises, la confusion est faite en catalan et en français entre "droits civils» et "droits civiques», dans la presse comme dans les discours. Qu'elle soit volontaire ou non, cette erreur de transposition d'un concept juridique d'un système institutionnel vers un autre révèle pourtant la difficulté à traduire l'intention du propos. Or, outre la nuance juridique entre droits civils et droits civiques, la charge de l'un ou l'autre terme est substantiellement différente. Si le locuteur parle de droits civils, il se place 
automatiquement dans le champ des valeurs et principes universels, ratifiés par l'Assemblée générale des Nations unies dans le Pacte international relatif aux droits civils et politiques adopté le 16 décembre 1966. En revanche, s'il renvoie à la défense des droits civiques des Catalans, il introduit une allusion aux luttes menées entre 1945 et 1970 par le Mouvement des droits civiques aux États-Unis pour obtenir le droit de vote des Noirs américains et mettre un terme à la ségrégation dans les États du Sud. Dans un cas, il s'agit de légitimer une cause en la plaçant sous l'égide d'une autorité universellement reconnue (ou presque), dans l'autre, d'exacerber le caractère discriminatoire d'un déni de reconnaissance de la légitimité de cette même cause. Dans les deux cas, on constate que les faits institutionnels permettant de capter et de retranscrire la charge du discours sont définis par les termes choisis par le locuteur et par le contexte dans lequel il souhaite s'inscrire de manière volontaire ou non. On voit également qu'ils peuvent être largement modifiés par le vocable retenu par le traducteur, altérant de ce fait la finalité et la portée du discours. II n'existerait donc pas de langue franche, non plus que d'éléments de langage, sans faits institutionnels, ni de faits institutionnels sans éléments de langage. Au traducteur de les maîtriser, CQFD?

Revenons au portrait-robot du parfait traducteur/diplomate, esquissé au début de notre propos. Le voici à présent affublé du costume de démiurge du discours, maniant l'art d'interpréter le doute et usant d'habiles litotes pour contourner les écueils d'une communication flove et ambiguë destinée à un auditoire diffus. Ce serait oublier que derrière son masque bifrons, le traducteur est à la fois critique et historien du discours, analyste et fin praticien des langages. II est, pour reprendre la comparaison de Jacques Catteau entre le traducteur et l'acteur, "à la fois spectateur froid et tranquille de son propre jeu, soumis sans cesse à l'analyse, et pourtant dispensateur de l'émotion la plus vraie».

s.arieslardeux@gmail.com 
Sabrina Ariès Lardeux est traductrice indépendante de l'espagnol et du catalan au français. Après avoir vécu et exercé dans la province de Gérone (Espagne) pendant dix ans, elle vient de s'installer en Aquitaine. S'intéressant particulièrement à la science politique et à la res publica d'un côté comme de l'autre de la frontière pyrénéenne, elle s'est spécialisée dans la traduction de la communication institutionnelle et politique.

\section{SOURCES CITÉES}

ARENDT Hannah, Du mensonge à la violence, Paris, Calmann-Lévy, 1972.

ARIFON Olivier, «Langue diplomatique et langage formel: un code à double entente», in Hermès, La Revue, 58, p. 69-78, 2010, www.cairn.info/revue-hermes-la-revue-2010-3-page-69. htm, consulté le 13 mai 2019.

BOUCHERON Patrick, DELALANDE Nicolas, «Récit national et histoire mondiale. Comment écrire l'histoire de France au xxI ${ }^{e}$ siècle? », in Histoire@Politique, 31, p. 17-26, www. cairn.info/revue-histoire-politique-2017-1-page-17.htm, consulté le 13 mai 2019.

CANAL Jordi, Con permiso de Kafka, el proceso independentista en Cataluña, Madrid, Atalaya, coll. «Península», 2018.

CATTEAU Jacques, «Les masques du traducteur», in Équivalences, 1-2, p. 7-18, 1991, www. persee.fr/doc/equiv_0751-9532_1991_num_20_1_1133, consulté le 13 mai 2019.

NOWICKI Joanna, OUSTINOFF Michaël, «La langue de bois, notion clé du monde contemporain ", in Hermès, La Revue, 71, 2015, p. 201-207, www.cairn.info/revue-hermes-la-revue2015-1-page-201.htm, consulté le 13 mai 2019.

OUSTINOFF Michaël, «Langues de bois d'hier et parler vrai d'aujourd'hui: de la "novlangue" aux "spin doctors" ", in Hermès, La Revue, 58, 2010, p. 15-21, www.cairn.info/revuehermes-la-revue-2010-3-page-15.htm, consulté le 13 mai 2019.

SEARLE John R., Les Actes de langage, essai de philosophie du langage, Paris, Hermann, Éditeurs des sciences et des arts, coll. «Savoir: Lettres», 1972. 